\title{
Surgeons, Especially Orthopedic Surgeons Should Know Fibromyalgia and Incomplete Form of Fibromyalgia
}

\section{Toda $\mathbf{K}^{*}$}

Department of Orthopedic Surgery, Kitahiroshima Town

Toyohira Hospital, J apan

*Corresponding author: Katsuhiro Toda, Department of Orthopedic Surgery, Kitahiroshima Town Toyohira

Hospital, 4705, Azaka, Kita-Hriroshima Town, YamagataGun, Hiroshima, 731-1222, J apan

Received: August 02, 2018; Accepted: September 12, 2018; Published: September 19, 2018

\begin{abstract}
Fibromyalgia (FM) and related syndrome (FMRS) often causes severe pain on any part of the body. This causes some kinds of problems. First, depending on the location of the pain, FMRS may be misdiagnosed with other diseases. In case that spinal surgery is performed, we should confirm that the cause of the symptoms is abnormality of the spine. Second, if patients with FMRS suffer from other painful diseases such as appendicitis, it is very difficult to find early other painful diseases. Surgeons have to make a final decision on the surgery. Third, surgeons often have to treat out patients. Treatment for spinal pain such as neck pain and low back pain without knowledge of FMRS is the same as talking about the American War of Independence without knowledge of George Washington. Forth, FM may be risk factor of complex regional pain syndrome. In case that patient with risk factor of complex regional pain syndrome undergo surgery, sufficient pain control is necessary.
\end{abstract}

Keywords: Fibromyalgia; Chronic widespread pain; Chronic regional pain; Complex regional pain syndrome; Lumbar canal stenosis; Central sensitization; Orthopedic surgeon

\section{Short Communication}

The prevalence of fibromyalgia (FM) is approximately $2 \%$ [1], and the prevalence of Chronic Widespread Pain (CWP) (incomplete form or early stage of FM) is approximately $10 \%$ [2], and the prevalence of Chronic Regional Pain (CRP) (incomplete form or early stage of CWP) is at least the prevalence of CWP worldwide [3]. The relative severities of the clinical symptoms in each diagnosis are FM $>$ CWP $>$ CRP [4]. In this article, FM, CWP, and CRP are collectively referred to as "FM and related syndrome (FMRS)"

FMRS often causes severe pain on any part of the body. This causes some kinds of problems. First, depending on the location of the pain, it may be misdiagnosed with other diseases. Second, if patients with FMRS suffer from other painful diseases such as appendicitis, it is very difficult to find early other painful diseases. Third, surgeons often have to treat out patients. Forth, FM may be risk factor of Complex Regional Pain Syndrome (CRPS) [5,6].

The first problem is more likely to occur in spinal surgery. In case of diagnosis of cervical disc herniation, lumbar disc herniation, and Lumbar Canal Stenosis (LCS), etc., differential diagnosis between spine diseases and FMRS is very important. Because the prevalence of FMRS is enormous, spine diseases are often associated with FMRS. In this case, diagnosis is very difficult, because symptoms are complicated. We have to make a diagnosis with the knowledge of typical symptoms of spine diseases and typical symptoms of FM. Based on the central sensitization theory, chronic pain causes central sensitization, regardless of the type of pain [7]. In other words, sensitized region in the brain and/or spinal cord causes pain. Therefore, spine diseases cause central sensitization. Stenosis of the lumbar canal on the image is different from LCS. Stenosis of the lumbar canal on the image is mere abnormality (stenosis) based on image such as X-ray, MRI, and
CT. It does not matter whether there is clinical symptoms or not. LCS is a clinical symptom such as intermittent claudication caused by stenosis of the lumbar canal on the image. If a patient with FMRS has stenosis of the lumbar canal on the image that does not cause clinical symptom, the patient is often misdiagnosed with LCS and undergoes unnecessary spinal surgery. Cervical disc herniation and lumbar disc herniation are in a similar state.

In Japan, many physicians believe that FM does not exist and orthopedic surgeons, especially spinal surgeons, are more likely to think that FM does not exist. When I showed my studies about FMRS at the medical congresses, orthopedic surgeons or ex-orthopedic surgeon alone asked questions saying that FM does not exist. Some patients with FMRS have been misdiagnosed with spine diseases and have undergone unnecessary spinal surgery. If unnecessary spinal surgery is performed, symptoms may be exacerbated.

Before spinal surgery is performed, we should confirm that the cause of the symptoms is abnormality of the spine. In order to do this, knowledge about FMRS (or FM) is necessary.

The second problem is very important in physicians who treat FMRS. Physicians who treat FMRS should recognize that pain of patients with FMRS is not always due to FMRS. Even if we know it, it is very difficult to find pain due to non-FMRS early in patients with FMRS. However, surgeons have to make a final decision on the surgery.

Surgeons often have to treat out patients. Especially, orthopedic surgeons and/or spinal surgeons may treat outpatient with neck pain and/or low back pain. Pharmacological treatment for FMRS [8] is very important for orthopedic surgeons and/or spinal surgeons. As mentioned above, the prevalence of FMRS is enormous. Treatment for FMRS is a mainstay of treatment for neuropathic pain in terms of 
prevalence, the number of treatment based on evidence, and diverse symptoms. Therefore, knowledge about FMRS is very important. Treatment for spinal pain such as neck pain and low back pain without knowledge of FMRS is the same as talking about the American War of Independence without knowledge of George Washington.

FM may be risk factor of CRPS [5,6]. FM has become an excuse for orthopedic surgeons who do not give patients with FM appropriate treatment in Japan. In other words, some orthopedic surgeons are reluctant to perform a surgery on patients with FM. This coping technique is not appropriate. It causes a major problem in case that surgery is necessary in an injured patient. For example, surgery is necessary for unstable displaced comminuted fracture of the distal end of the radius. Female sex [5,9-13] and headache or migraine $[12,14,15]$ also may be a risk factor of CRPS. Orthopedic surgeons who are reluctant to perform a surgery on patients with FM should be reluctant to perform a surgery on female patients or patients with headache or migraine. In case that patient with risk factor of CRPS undergo surgery, sufficient pain control is necessary. Although surgeons are responsible for an inappropriate treatment for CRPS, surgeons are not responsible for the occurrence of CRPS after surgery.

In conclusion, surgeons, especially orthopedic surgeons should know FMRS and perform appropriate coping technique.

\section{References}

1. Heidari F, Afshari M, Moosazadeh M. Prevalence of fibromyalgia in general population and patients, a systematic review and meta-analysis. Rheumato Int. 2017; 37: 1527-1539.

2. Andrews $P$, Steultjens M, Riskowski J. Chronic widespread pain prevalence in the general population: A systematic review. Eur J Pain. 2018; 22: 5-18.

3. Toda K, Harada T. Prevalence, classification, and etiology of pain in Parkinson's disease: association between Parkinson's disease and fibromyalgia or chronic widespread pain. Tohoku J Exp Med. 2010; 222: 1-5.

4. Toda K. Comparison of symptoms among fibromyalgia syndrome, chronic widespread pain, and an incomplete form of chronic widespread pain. J Musculoskelet Pain. 2011; 19: 52-55.
5. Lipman MD, Hess DE, Werner BC, Deal DN. Fibromyalgia as a Predictor of Complex Regional Pain Syndrome After Distal Radius Fracture. Hand (NY). 2017.

6. Crijns TJ, van der Gronde B, Ring D, Leung N. Complex Regional Pain Syndrome after Distal Radius Fracture Is Uncommon and Is Often Associated with Fibromyalgia. Clin Orthop Relat Res. 2018; 476: 744-750.

7. Schaible HG. Mechanisms of Chronic Pain in Osteoarthritis. Curr Rheumato Rep. 2012; 14: 549-556.

8. Toda K. The Medication Guidelines for Neuropathic Pain Including Fibromyalgia is justified from a Scientific Viewpoint; however, it does Not Necessarily Agree with Clinical Priority. Fibromyalgia: Open Access. 2018; 3: 1000128.

9. de Mos M, de Bruijn AG, Huygen FJ, Dieleman JP, Stricker BH, Sturkenboom MC. The incidence of complex regional pain syndrome: A population-based study. Pain. 2007; 129: 12-20.

10. Jellad A, Salah S, Frih ZB. Complex regional pain syndrome type I. Incidence and risk factors in patients with fracture of the distal radius. Arch Phys Med Rehabil. 2014; 95: 487-492.

11. Pons T, Shipton EA, Williman J, Mulder RT. Potential risk factors for the onse of complex regional pain syndrome type 1: a systematic literature review. Anesthesiol Res Pract. 2015; 2015: 956539.

12. Elsharydah A, Loo NH, Minhajuddin A, Kandil ES. Complex regional pain syndrome type 1 predictors - Epidemiological perspective from a national database analysis. J Clin Anesth. 2017; 39: 34-37.

13. Petersen PB, Mikkelsen KL, Lauritzen JB, Krogsgaard MR. Risk factors for post-treatment complex regional pain syndrome (CRPS) - an analysis of 647 cases of CRPS from the Danish Patient Compensation Association. Pain Pract. 2018; 18: 341-349.

14. Toda $\mathrm{K}$, Muneshige $\mathrm{H}$, Maruishi M, Kimura $\mathrm{H}$, Asou T. Headache may be a risk factor for complex regional pain syndrome. Clin Rheumatol. 2006; 25 : 728-730

15. de Mos M, Huygen FJ, Dieleman JP, Koopman JS, Stricker BH, Sturkenboom MC. Medical history and the onset of complex regional pain syndrome (CRPS). Pain. 2008; 139: 458-466.
Austin J Surg - Volume 5 Issue 7 - 2018

ISSN : 2381-9030 | www.austinpublishing group.com

Toda. (C) All rights are reserved
Citation: Toda K. Surgeons, Especially Orthopedic Surgeons Should Know Fibromyalgia and Incomplete Form of Fibromyalgia. Austin J Surg. 2018; 5(7): 1148 